\title{
The Use of Iliac J-Bone Block for Three Dimensional Correction of
} Alveolar Defect

\author{
Kweijing Chen ${ }^{1}$, Edward Chengchuan $\mathrm{Ko}^{2-4}$, Li Jih Fuh ${ }^{5,6}$ and Michael Yuanchien Chen ${ }^{1,6 *}$
}

${ }^{1}$ Department of Dentistry, Division of Oral and Maxillofacial Surgery, China Medical University Hospital, China Medical University, Taichung City, Taiwan ${ }^{2}$ Department of Oral and Maxillofacial Surgery, Kaohsiung Medical University Hospital, Kaohsiung City, Taiwan

${ }^{3}$ School of Dentistry, College of Dental Medicine, Kaohsiung Medical University, Kaohsiung City, Taiwan

${ }^{4}$ Department of Cartilage and Bone Regeneration (Fujisoft), Graduate School of Medicine, University of Tokyo, Tokyo, Japan

${ }^{5}$ Department of Dentistry, Division of Prosthodontics, China Medical University Hospital, Taichung City, Taiwan

${ }^{6}$ School of Dentistry, College of Medicine, China Medical University, Taichung City, Taiwan

\begin{abstract}
This study aimed to correct complicated alveolar defects with autogenous iliac bone blocks, thereby facilitating subsequent dental implant-supported restorations. Using a retrospective study design, 27 consecutive patients (age, 1963 years) who underwent iliac "J-bone block" procedures from 2006 through 2010 were enrolled. All the reconstruction materials were harvested from the superior and medial aspects of the anterior iliac crest. Each iliac bone block was tailored to passively fit the contour of the alveolar defect and secured in position by titanium mini-screws to achieve significant bone volume expansion in both the transverse and vertical dimensions. Pre and post-operative computed tomography imaging studies with a radiographic guide in position were conducted to evaluate the changes in shape and volume of the recipient sites. All iliac bone grafts healed uneventfully with minimal volume shrinkage and unremarkable donor site morbidity. A total of 73 dental implants in 21 patients appeared clinically healthy. Iliac cortico-cancellous bone blocks were easily manipulated by hand instruments and transformed into a J-bone block, which lead to high-quality bone augmentation and allowed ideal implant positioning with proper axial alignment. We suggest that iliac J-bone block be used in the near future to improve the success rate of complicated implant restorations.
\end{abstract}

Keywords: J-bone block; Iliac bone grafting; Complicated alveolar defect

\section{Introduction}

Technological advances over the past three decades have increased the usefulness and efficiency of dental implants. However, successful prosthesis implantation requires that it be fastened to the patient's own alveolar ridge, which must be of sufficient height and width, and have an appropriate appearance and soft tissue conditions. Long-term tooth loss, trauma, jaw-bone infection, and tumor resection can all cause varying degrees of alveolar ridge defects in patients. Therefore, bone graft surgery is suggested as the first step in the reconstruction process of the oral cavity for this group of patients.

Bone graft materials specifically suited to the needs of individual patients are available. These materials can be divided into 4 categories namely autogenous bone grafts, alloplastic graft materials, allografts and xenografts.

The main advantage of autogenous bone grafts is that living boneproducing cells can be planted at the site of the bone deficit, causing shortening of the healing time and formation of relatively high-quality new bone. For large bone defect areas, it is definitely the best choice of reconstruction material. Frequently used sources of autogenous bone grafts include the skull, jaw bones, ribs, ilium, and fibula, of which the iliac and tibial cancellous bones are most commonly used. In addition, large amounts of cortical bone can be retrieved from the ilium to support the soft tissue flap tension. The ilium is especially useful in complex cases of alveolar ridge defect.

There is a large amount of English-language literature available on alveolar ridge reconstruction, most of them aiming to improve the technique of raising the alveolar ridge height. In fact, increasing and maintaining the height of the alveolar ridge has always been a difficult challenge for reconstruction physicians. Traditional guided bone graft regeneration is more suitable in cases of smaller defects. For patients who also have less alveolar bone width due to atrophy, fixing the piece of bone directly to the receiving bone area (onlay block grafting) is associated with a risk of graft failure due to poor fixation.

In 2007, Pelo et al. improved sandwich osteotomy method proposed by Jensen et al., and combined it with onlay bone grafting, simultaneously increasing the height and width of the front alveolar ridge, but the technical requirements for setting the bone were very stringent [1,2]. Later in 2008, Louis et al. used a titanium mesh to support the shape of the soft tissue, the space between the metal mesh and the alveolar bone being filled with iliac cancellous bone [3]. Notwithstanding a bone graft success rate of $97 \%$, this method was associated with a $52 \%$ risk of titanium mesh exposure in the oral cavity post-surgery. Of note, early exposure will greatly affect the final results of bone graft surgery. In 2010, Pelo et al. improved on the method of interpositioning of alveolar bone grafts for treatment of patients with large areas of alveolar ridge atrophy [4]. According to their report, 19 patients underwent horizontal osteotomy of the mandible: a piece from the iliac bone was inserted in the area of surgery, and then a titanium plate was used to fix the iliac bone. Of the 141 Biomet 3 I OSSEOTITE $^{\circ}$ dental implants inserted at the recipient site, $90 \%$ were implanted successfully. Nonetheless, this method only increases the height of the alveolar ridge, and thus is not applicable when there is the need to

*Corresponding author: Dr. Michael Yuanchien Chen, Chief, Department of Dentistry, Division of Oral and Maxillofacial Surgery, China Medical University Hospital, No. 2, Yu-der Road, Taichung City, Taiwan, Tel: +886 975681380; E-mail: mychen@mail.cmuh.org.tw

Received January 20, 2016; Accepted February 12, 2016; Published February 19, 2016

Citation: Chen K, Ko EC, Fuh LJ, Chen MY (2016) The Use of lliac J-Bone Block for Three Dimensional Correction of Alveolar Defect. Dentistry 6: 363 doi:10.4172/2161-1122.1000363

Copyright: $\odot 2016$ Chen K, et al. This is an open-access article distributed under the terms of the Creative Commons Attribution License, which permits unrestricted use, distribution, and reproduction in any medium, provided the original author and source are credited. 
increase the width. Additionally, if applied to cases of severe atrophy, there is a risk of mandible fracture.

In this paper, we describe an autogenous iliac J-bone block method. This method can be applied in the treatment of complex cases where the alveolar ridge is deficient in both height and width. In our hands, it has already been successfully applied to 27 cases of alveolar ridge reconstruction in both the anterior and posterior tooth areas.

\section{Materials and Methods}

From 2006 to 2010, 27 patients (11 male and 16 female) underwent alveolar defect reconstruction treatment with autogenous iliac J-bone block. Their ages ranged from 19 to 63 years, with a mean of 41.3 years (Table 1). All patients accepted the bone graft surgery in the hope of restoring occlusal function with the help of dental implants. All 27 patients were operated on by the same oral and maxillofacial surgeon. The procedure first involved extracting bone blocks and cancellous bone from the iliac bone, and thereafter proceeding with jaw-bone reconstruction.

Before surgery, the patients' medical history was reviewed. In addition, a front hip X-ray was taken to exclude patients with iliac lesions or previous surgery. Before undergoing alveolar bone reconstruction, all patients wore a radiographic guide to be used during the computed tomography (CT) scan. The CT scan allows estimation of the size and number of bone blocks needed for jaw-bone reconstruction.

\section{Surgical procedures at donor site}

Surgery proceeded with patients under general anesthesia in the supine position (Figure 1). The bone material needed for reconstruction was taken from the inner part of the anterior-superior iliac crest. First, the patient's hip area was raised up with a cloth-covered mattress, causing the anterior superior iliac crest to protrude, and making it easy for the surgeon to confirm its location. After skin disinfection, a colored pen was used to sketch the outline of the anterior-superior iliac crest (Figure 1A) $2 \mathrm{~cm}$ lateral to the iliac crest [5], starting at $1 \mathrm{~cm}$ behind the anterior-superior iliac spine to avoid the main sensory nerve (lateral cutaneous branch of the iliohypogastric nerve and femoral cutaneous nerve); after cutting through the dermis and subcutaneous fat tissue, the periosteum was exposed. An incision was made in the periosteum along the outer curvature of the iliac crest, and was then extended inward on 2 sides. Such a design facilitates surgical operation since it exposes a relatively large donor site. Then, the anterior superior iliac crest and the ventral cortical bone were exposed using a sharp periosteal dissector, while care was taken to avoid subperiosteal dissection, preventing damage to the deep circumflex iliac artery [6]. While carefully protecting the surrounding soft tissue, an electric microsaw was used to cut out the required number of donor bones, with the required size (Figure 1B). The cortico-cancellous bone block was then carefully removed with an osteotome (Figure 1C). A semicircular gouge and a rongeur were used to extract a copious quantity of cancellous bone (Figure 1D). During the bone extraction process, the posterior cortical bone was kept intact. The required amount of cancellous bone was retrieved using a suction tip with a filter. After controlling the bleeding at the donor site with the proper amount of bone wax (Johnson \& Johnson), the incision was filled with gelfoam (Johnson \& Johnson), and 4-0 Vicryl was used to seal shut the periosteum and the subcutaneous tissue. Then, 6-0 Nylone was used to suture the skin. The wound was covered with gauze, which was pressed against the wound for 1 week after surgery. Patients could get out of bed the first day after surgery, but were prohibited from taking part in any strenuous exercise for the following 3 months, especially activities that require a sudden generation of force from the hip/buttocks area, such as running, hiking, and playing golf.

\section{Surgical procedures at recipient site}

After carefully peeling off the soft tissue flap, slight repairs were made to the irregular shape of the alveolar ridge to facilitate a bone

\begin{tabular}{|c|c|c|c|c|c|c|}
\hline No. & Gender & Age & $\begin{array}{c}\text { Date of } \\
\text { operation }\end{array}$ & $\begin{array}{l}\text { Surgical } \\
\text { site }\end{array}$ & $\begin{array}{c}\text { Source of } \\
\text { defect }\end{array}$ & $\begin{array}{c}\text { No. of } \\
\text { implants }\end{array}$ \\
\hline 001 & $\mathrm{~F}$ & 44 & $2006 / 12 / 22$ & UL & PR & 0 \\
\hline 002 & M & 49 & $2007 / 06 / 27$ & UB & PR & 7 \\
\hline 003 & $\mathrm{~F}$ & 49 & $2007 / 08 / 15$ & UL & PR & 0 \\
\hline 004 & $\mathrm{~F}$ & 22 & 2007/10/19 & UL & PR & 0 \\
\hline 005 & $\mathrm{~F}$ & 39 & $2008 / 03 / 28$ & LL & BT & 4 \\
\hline 006 & $\mathrm{~F}$ & 19 & $2008 / 07 / 04$ & UA & $\mathrm{Tr}$ & 0 \\
\hline 007 & M & 20 & $2008 / 07 / 30$ & UR & PR & 3 \\
\hline 008 & M & 21 & $2008 / 08 / 06$ & UA & PR & 3 \\
\hline 009 & M & 48 & $2008 / 09 / 19$ & UL & PR & 3 \\
\hline 010 & M & 28 & $2008 / 09 / 19$ & UA & $\mathrm{Tr}$ & 3 \\
\hline 011 & $\mathrm{~F}$ & 48 & $2008 / 10 / 24$ & UB & $\mathrm{Tr}$ & 7 \\
\hline 012 & $\mathrm{~F}$ & 37 & $2008 / 11 / 21$ & UA & PR & 2 \\
\hline 013 & $\mathrm{~F}$ & 23 & $2008 / 11 / 26$ & UA & $\mathrm{Tr}$ & 4 \\
\hline 014 & $\mathrm{~F}$ & 37 & $2008 / 12 / 03$ & UR & PR & 5 \\
\hline 015 & M & 25 & $2009 / 01 / 02$ & LA & $\mathrm{Tr}$ & 3 \\
\hline 016 & M & 59 & $2009 / 03 / 04$ & UB/LA & PR & 0 \\
\hline 017 & $\mathrm{~F}$ & 53 & $2009 / 03 / 13$ & UB & PR & 8 \\
\hline 018 & $\mathrm{~F}$ & 47 & $2009 / 03 / 27$ & UA & PR & 4 \\
\hline 019 & $\mathrm{~F}$ & 44 & $2009 / 05 / 29$ & UA & PR & 3 \\
\hline 020 & M & 44 & $2009 / 06 / 24$ & UL & PR & 2 \\
\hline 021 & $\mathrm{~F}$ & 49 & $2009 / 07 / 03$ & UA & PR & 3 \\
\hline 022 & M & 54 & $2009 / 11 / 26$ & UA & PR & 0 \\
\hline 023 & M & 55 & $2010 / 01 / 14$ & LR & PR & 2 \\
\hline 024 & M & 40 & $2010 / 06 / 03$ & UR & PR & 2 \\
\hline 025 & $\mathrm{~F}$ & 44 & $2010 / 08 / 11$ & UR & PR & 0 \\
\hline 026 & $\mathrm{~F}$ & 56 & $2010 / 10 / 08$ & UA & PR & 3 \\
\hline 027 & $\mathrm{~F}$ & 63 & $2010 / 10 / 29$ & UR & PR & 2 \\
\hline
\end{tabular}

UA: upper anterior; LA: lower anterior; UB: upper full arch; UR: right upper arch; UL: left upper arch; LL: left lower arch; PR: physiologic resorption; Tr: trauma; BT: benign tumor

Table 1: Characteristics of the patients enrolled in the study

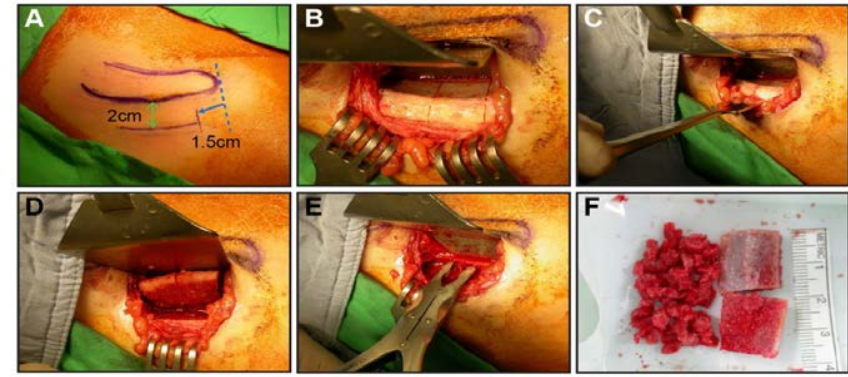

A: A skin incision line was made $2 \mathrm{~cm}$ lateral to the anterior iliac crest and $1.5 \mathrm{~cm}$ behind the anterior superior iliac spine, avoiding the lateral cutaneous branch of the iliohypogastric nerve and the femoral cutaneous nerve; $B$ : Microsaws were used to cut out the right amount and size of bone blocks from the donor site; C,D: A flat osteotome was used to carefully remove the cortico-cancellous bone blocks; E,F: A copious quantity of cancellous bone was retrieved from the interior ilium with a rongeur and a semicircular gouge; ASIS: anterior superior iliac spine.

Figure 1: Surgical procedures at donor site. 
block fit; a small round bur was eventually used in the thick cortical bone area to create several bleeding points, which facilitate the union of the bone graft with the recipient site (Figure 2). The rongeur was used to shape the previously removed cortico-cancellous bone block, and to fasten it stably in the recipient site, thus increasing the height and width of the alveolar ridge (Figure 2B). Viewed from the side, the bone block has the shape of the letter "J" of the classical Latin alphabet (Figure 2C), for which we named it "iliac J-bone block". Each bone block was fastened with 2 miniscrews (Leibinger). Any sharp edges of the bone were carefully rounded, and the gap between the surrounding tissue of the bone block and recipient site was filled in with cancellous bone. The soft tissue was then sutured together after proper tension relief treatment. The patients were asked not to wear removable temporary dentures for 1 month, so as not to inflict the surgical area with inappropriate biting force, which would not be beneficial for graft healing.

\section{Results}

In total, 27 patients underwent alveolar ridge reconstruction. The average number of days in hospital after surgery was 2.16. All of the patients were able to get up and walk on the first day after surgery. Overall, there were limited postoperative complications in the donor area (Table 2). In addition to postoperative wound pain and bruising, there was one occurrence of subcutaneous hematoma, which healed after the blood was suctioned out with a needle, and the site was subjected to continuous compression for a week. No severe swelling, numbness of skin surrounding the wound, infections, unsightly scars, or other complications was noted. Under this careful surgical procedure, there were also no cases of major complications such as abdominal puncture. Donor site wound pain assessment was based on a visual analog scale. On a scale from 0 (not painful) to 100 (extremely painful), the average score for pain in the lower extremities of patients when subjected to stress induced by carrying a weight on the first day after surgery was 43.9 , but had declined to 18.3 by the day of discharge. On the other hand, the pain induced when patients exerted their abdominal muscles (such as when coughing, sneezing, or laughing) was significantly greater than the pain caused by carrying a weight.

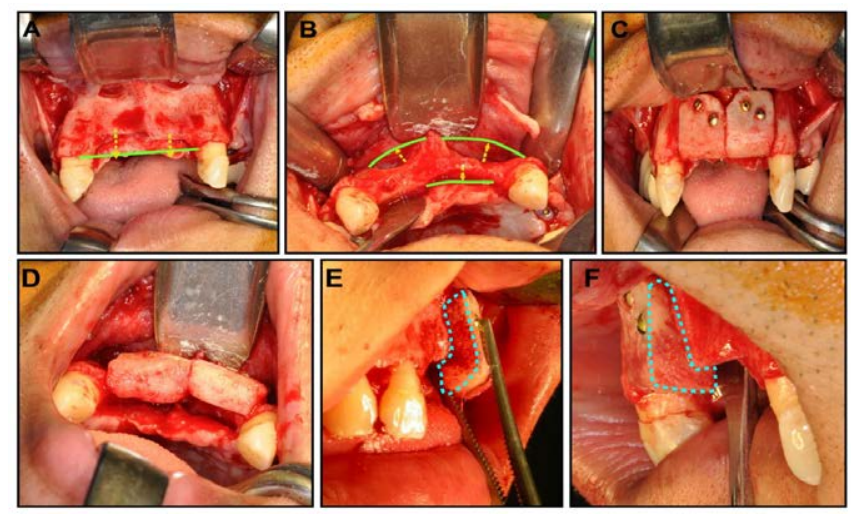

A,B: A case of severe horizontal and vertical upper-jaw front-tooth alveolar bone atrophy caused by long-term tooth loss; C,D: The sponge bone block retrieved from the iliac cortical bone was fixed to the recipient site with 2 miniscrews, thus increasing alveolar bone height and width. The screw head was deliberately trimmed down to the level of the outer edge of the bone graft to facilitate future evaluation of volume shrinkage; E,F: Due to the similarity of the bone block to the letter "J" when viewed from the side, we named it "J-bone block."

Figure 2: Surgical procedures at recipient site.

\begin{tabular}{|c|c|}
\hline Complication & Number of cases (\%) \\
\hline Gait disturbance (3 months) & 0 \\
\hline Iliac wing fracture & 0 \\
\hline Unpleasant scar & 0 \\
\hline Ecchymosis & $1(4.5 \%)$ \\
\hline Hematoma & $1(4.5 \%)$ \\
\hline Infection & 0 \\
\hline Abdominal entry & 0 \\
\hline Temporary skin paresthesia & $6(27.2 \%)$ \\
\hline
\end{tabular}

Table 2: Morbidity and complication rates at donor site.

Nonetheless, 1 month after the procedure, the pain did not significantly affect the activities of daily life.

As for the recipient site, postoperative infections and wound dehiscence did not occur. Only in one case, a small piece of dead bone was found 3 weeks after the implantation. The piece fell off but did not have any adverse effects on the implant prognosis. At the time of preparing this manuscript, a total of 21 patients had undergone dental implant surgery. The surgery was carried out an average of 6.5 months after reconstructive surgery, and a total of 73 dental roots had been implanted in the recipient area (Table 1). In the following paragraphs, we report the complete course of treatment in two cases in which functionality was retained after reconstruction.

\section{Case 1}

A 25-year old female patient who lost her right and left upper middle incisors, upper left incisor, and canines presented with serious front upper alveolar ridge atrophy due to trauma. The patient hoped that an implant combined with fixed-type bridges could restore her chewing function and esthetics. The CT scan revealed the frontal upper alveolar ridge height and width to be clearly insufficient for dental implant. For this reason, the autogenous iliac J-bone block reconstruction method was chosen (Figure 3). Six months after reconstructive surgery, dental implant surgery commenced, and after about a year, the entire reconstruction process was complete. The size and shape of the crowns were all within reasonable range (Figure $3 \mathrm{~L}$ ).

\section{Case 2}

A 40-year old woman with an ameloblastoma in the left mandibular body underwent tumor resection and peripheral bone trimming in December 2005 under general anesthesia, conducted with the aim of speeding up the new bone generation process in the lower jaw as soon as possible, to reduce pathological fracture risk. For reconstruction using autogenous bone grafts, $15 \mathrm{~cm}^{3}$ of cancellous bone was retrieved from the patient's right tibia (Figure 4). As there was no tumor recurrence after 3 years, the patient wanted to undergo implant surgery to restore chewing function. Because of the previous tumor resection, however, the left lower jaw alveolar ridge height and width were not sufficient for inserting dental implants of the ideal length and diameter. Moreover, there was insufficient vestibular depth, and keratinized gingival court shortage. Therefore, an autogenous iliac J-bone block reconstruction surgery was arranged for March 2008. Soft tissue reconstruction surgery including vestibuloplasty and keratinized gingiva grafting was performed in August 2008. In November 2008 (about 8 months after bone graft surgery), 4 dental implants were implanted in the left lower jaw, successfully completing the prosthodontic treatment (Figure $4 \mathrm{G}, \mathrm{H})$. During soft tissue reconstruction prior to the implant surgery, it was confirmed that the patient's oral vestibule had the proper depth, and that there was sufficient keratinized gum around the implant. The 


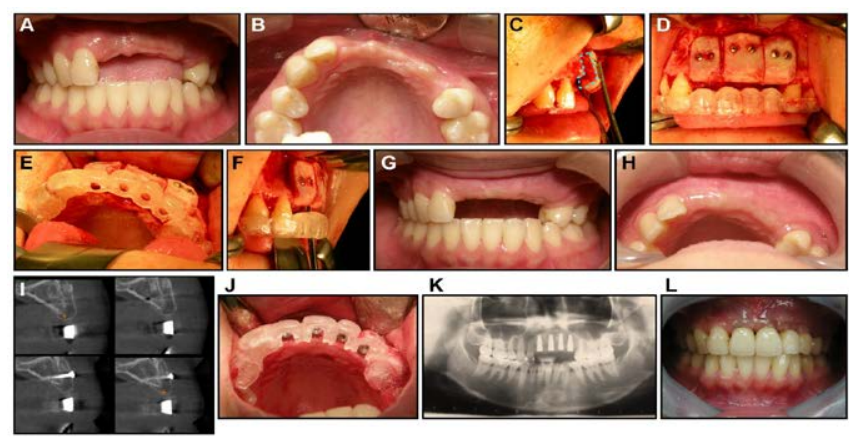

$A, B$ : A patient with trauma-induced loss of the upper left and right middle incisor, side incisors, and canine teeth; C: Reconstruction of alveolar bone height and width by using the autogenous iliac J-bone block method; D,E: A surgical guide was used to confirm that the increase in height and width was adequate. All of the bone blocks were fixed with 2 miniscrews; $F$ : The height and width of the alveolar ridge were sufficient according to dentistry ideals for position and angle of dental implants; G,H: Five months after surgery, the alveolar ridge was quite dense and the alveolar ridge arc was quite normal; I: In this pre-dental implant surgery computed tomography scan image showing a patient wearing a radiographic guide, the bone graft is still visible. The bone graft volume was stable with negligible horizontal shrinkage; J: Four dental implants were inserted in full accordance with the position of the surgical guide. In each case, the width and height of the alveolar bone was sufficient to allow the ideal implant length and diameter; K: An X-ray taken after dental implant surgery shows four implants with good inter-implant distance and parallelism; L: After completing the prosthodontic treatment, the positions of the crowns were the ideal, and their size and length were suited for restoring the patient's chewing function as well as esthetics.

Figure 3: Case 1.

latter is necessary from the viewpoint of oral hygiene, especially that of the implant.

\section{Discussion}

There is strong evidence supporting the long-term efficacy and safety of dental implants [7]. Different types of bone substitutes can be used for alveolar bone remodeling. However, for areas of large bone loss, autogenous bone grafting is the best choice because of its good osteogenic potential in autogenous bone. The amount of cancellous bone that can be retrieved from the upper front part of the ilium is about the same as that can be retrieved from the proximal tibia parts (Figure 3) [8]. However, the cases herein discussed combine the complexity of both horizontal and vertical alveolar bone atrophy. Hence, the procedures required the retrieval of not only cancellous bone, but also of cortico-cancellous bone blocks. This is the reason as to why the upper front iliac crest was the most appropriate source in the present cases. Iliac bone blocks can be taken from either the anterior or posterior ilium. Although the largest amount of bone can be retrieved from the posterior ilium [9], the amount of bone retrieved from the anterior ilium is typically sufficient. By taking bone from the anterior ilium, we also avoid having to change the patient's posture during surgery.

An anterior iliac bone retrieval approach can in turn be divided into 2 different approaches: anteriomedial and anteriolateral. Our approach was the anteriomedial one. The process involves first cutting through the skin and the subcutaneous tissue layer, and then cutting the periosteum, peeling it inwards while protecting the periosteum and avoiding irritation of the iliacus muscle and deep circumflex iliac artery. The iliac crest lateral muscles are not peeled off, keeping the tensor fasciae latae muscle attachment region intact [6]. The tensor fasciae latae muscle controls the abduction and flexion of the legs; maintaining its integrity can reduce postoperative pain when walking, avoid gait disturbance, and shorten recovery.

Of 27 cases, 21 patients received subsequent dental implant reconstruction at an average of 6.5 months after reconstructive bone graft surgery. Although some studies $[10,11]$ have indicated that implanting artificial teeth directly at the time of reconstructive surgery can shorten the course of treatment, others [12-14] conversely believe that the success rate is higher for a two-stage approach than for a onestage approach. If the contact area between the transplanted bone and the recipient site has already begun to heal at the time of dental implant surgery, the osseointegration mechanisms will be similar to those for regular non-implant bone. In line with this thought, the degree of integration between the implant and the bone graft should be superior to that of natural bone [15]. In addition, the requirements in implant-supported fixed prosthesis with regard to position and angle are generally higher. This is especially important for the aesthetic appearance of the upper jaw area, and careful evaluation should be conducted. It is preferable not to proceed with dental implant surgery until 3-6 months after bone graft surgery, by which time the bone remodeling process will have stabilized.

Regardless of the type of bone used as reconstruction material, volume shrinkage will occur. Volume shrinkage has been described to be smaller in membranous bone than in endochondral bone [16,17]. This may be because the membranous bone has a higher density

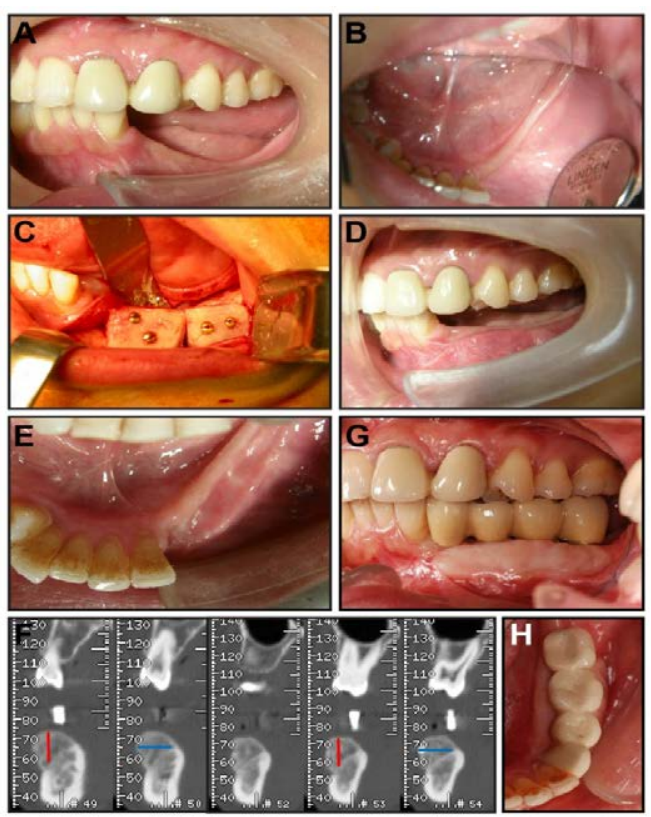

A,B: A 40-year-old female patient with inadequate height and width of the alveolar bone, inadequate vestibular depth, and a lack of keratinized gingiva due to tumor cell resection; C: Autogenous iliac J-bone block reconstruction of alveolar bone height and width; D,E: About 5 months after bone graft surgery, alveolar bone height and width were quite adequate and stable, albeit obvious lack of keratinized attached gingiva; F: Pre-implant computed tomography scans obtained before dental implant surgery showing the shape of the bone graft with significant increase of the bone mass, both vertically and horizontally. The distance between the bone graft and the Leibinger microscrew indicates that volume shrinkage was very small; $\mathrm{G}, \mathrm{H}$ : After completing the prosthodontic treatment, the crowns were of reasonable size, length, and position. The patient's chewing function was successfully restored.

Figure 4: Case 2. 
of cortical bone, and cortical bone maintains its volume better than cancellous bone does. Other contributing factors could be the degree of vascularization during the healing process; the damage afflicted on the bone graft during surgery; whether or not the bone graft has been properly immobilized; poor healing of soft tissue wounds in the oral cavity, leading to early exposure of the bone graft or wound dehiscence; and whether or not regular adjustments of temporary dentures were made before dental implant surgery, to avoid adding inappropriate pressure to the bone graft area [18]. In the present study, we used CT scan images (Figure 5) taken prior to dental implant surgery, and observed the degree to which the miniscrews protruded from the bone graft made during dental implant surgery (Figure 6), to assess the extent of graft bone volume shrinkage. CT scan images show small bone graft volume shrinkage. The shape of the bone graft was still distinguishable, and the screw head of the miniscrew immobilizing the bone block was leveled with the outer edge of the ilium bone block. When a soft tissue flap was opened during implant surgery, it was confirmed that the protrusion of the screw head was less than $1 \mathrm{~mm}$ in almost all cases. From these results and the available literature, it can be established iliac bone graft material can be quite stable if handled properly [18,19].

Currently, autogenous bone grafting is the gold standard in managing large alveolar ridge defect. Autogenous bone grafts from extra-oral donor sites present intraoperative disadvantages and postoperative complications. Since 2002, US Food and Drug Administration (FDA) has approved the use of absorbable collagen sponge (ACS) as a carrier for recombinant human BMP-2 (rhBMP-2) in lumbar fusion and long bone fracture repairs. In 2007, rhBMP-2 was used for alveolar ridge and maxillary sinus augmentation [20]. The introduction of rhBMP technology has raised the prospect of predictable bone regeneration and dental implant osseointegration. [21,22]. However the lack of suitable delivery systems has hampered the clinical use of rhBMP-2 [23]. In 2015, Filho et al. used titanium mesh and rhBMP-2 for the repair of major bone defects in the alveolar bone. CBCT scans confirmed that this method provided effective augmentation of the atrophic anterior maxilla prior to implant placement. But all the patients had developed D4 quality bone by the start of implant placement [24]. Minimal resistance to drilling was observed. Similarly, Misch reported that de novo bone induction by rhBMP-s requires a longer period of time for mineralization to occur [25]. The quality of bone

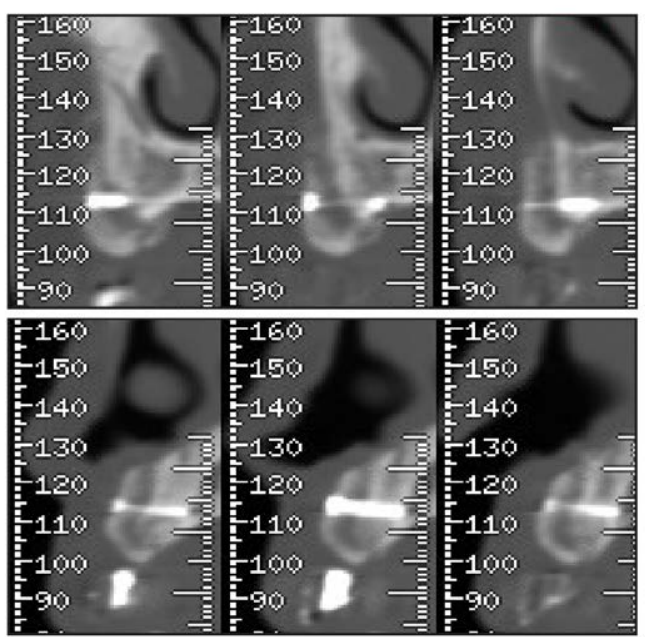

Figure 5: Computed tomography scan images taken 6 months after surgery showing the shape of the bone grafts, with stable height and width.

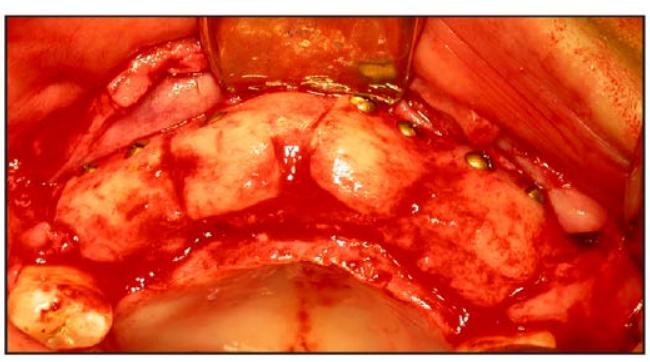

When the skin flap was re-opened, actual observations and measurements showed minimal volume shrinkage of the transplanted bone grafts.

Figure 6: Dental implant surgery was performed at an average of 6.5 months after bone graft surgery.

was compromised. The use of rhBMP-2 with various carriers should be confirmed by more clinical trials that includes a long observation period and a larger sample sizes.

Treatment combined vertical and horizontal alveolar ridge atrophy by means of autogenous iliac J-bone block has the following advantages. It increases both the height and width of the alveolar ridge, it is easily shaped to fit the recipient site by use of a rongeur, it is easily immobilized into place by using just one miniscrew in the center of the contact area, it maintains the bone graft volume, the cancellous bone used to fill the surrounding area can promote early revascularization, a stable volume of the grafted bone after surgery can provide the alveolar bone with the width and height required for later dental implant surgery, it is beneficial for reconstruction of both the anterior or posterior tooth region, in the upper or lower jaw alveolar bone and when performed properly, complications at the donor site are limited.

\section{Conclusion}

Altogether, autogenous iliac J-bone block technique provides a highly predictable outcome of severe alveolar ridge atrophy where 3-D reconstruction is performed before dental implant surgery. We suggest that iliac J-bone block be used in the future to improve the success rate of complicated implant restorations.

\section{References}

1. Pelo S, Boniello R, Moro A, Gasparini G, Amoroso PF (2010) Augmentation of the atrophic edentulous mandible by a bilateral two-step osteotomy with autogenous bone graft to place osteointegrated dental implants. Int $\mathrm{J}$ Oral Maxillofac Surg 39: 227-234.

2. Jensen OT, Kuhlke L, Bedard JF, White D (2006) Alveolar segmental sandwich osteotomy for anterior maxillary vertical augmentation prior to implant placement. J Oral Maxillofac Surg 64: 290-296.

3. Louis PJ, Gutta R, Said-Al-Naief N, Bartolucci AA (2008) Reconstruction of the maxilla and mandible with particulate bone graft and titanium mesh for implant placement. J Oral Maxillofac Surg 66: 235-245.

4. Pelo S, Boniello R, Gasparini G, Longobardi G, Amoroso PF (2007) Horizontal and vertical ridge augmentation for implant placement in the aesthetic zone. Int J Oral Maxillofac Surg 36: 944-948.

5. Nkenke E, Weisbach V, Winckler E, Kessler P, Schultze-Mosgau S, et al. (2004) Morbidity of harvesting of bone grafts from the iliac crest for preprosthetic augmentation procedures: A prospective study. Int J Oral Maxillofac Surg 33: 157-163.

6. Marx RE (2005) Bone harvest from the posterior ilium. Atlas Oral Maxillofac Surg Clin North Am 13: 109-118.

7. Albrektsson T, Zarb G, Worthington P, Eriksson AR (1986) The long-term efficiency of currently used dental implants: a review and proposed criteria of success. Int J Oral Maxillofac Implants 1: 11-25. 
Citation: Chen K, Ko EC, Fuh LJ, Chen MY (2016) The Use of Iliac J-Bone Block for Three Dimensional Correction of Alveolar Defect. Dentistry 6: 363. doi:10.4172/2161-1122.1000363

8. Chen MY, Ko EY, Fuh LJ (2005) Modified autogenous tibial cancellous bone grafting: Applications in jaw bone reconstruction. Chin J Oral Maxillofac Surg 16: 36-47.

9. Marx RE, Morales MJ (1988) Morbility from bone harvest in major jaw reconstruction: a randomized trial comparing the lateral anterior and posterior approaches to the ilium. J Oral Maxillofac Surg 46: 196-203.

10. Marx RE, Morales MJ (1998) The use of implants in the reconstruction of oral cancer patient. Dental Clinics North Am 42: 177-202.

11. Misch CM (1997) Comparison of intraoral donor sites for onlay grafting prior to implant placement. Int J Oral Maxillofac Implants 12: 767-776.

12. Sjostrom M, Lundgren S, Sennerby L (2006) A histomorphometric comparison of the bone graft - titanium interface between interpositional and onlay/inlay bone grafting technique. Int J Oral Maxillofac Implants 21: 52-62.

13. Reinert S, König S, Bremerich A, Eufinger H, Krimmel M (2003) Stability of bone grafting and placement of implants in the severely atrophic maxilla. $\mathrm{Br} \mathrm{J}$ Oral Maxillofac Surg 41: 249-255.

14. Verhoeven JW, Cune MS, Ruijter J (2006) Permucosal implants combined with iliac crest onlay grafts used in extreme atrophy of the mandible: Long-term results of a prospective study. Clin Oral Implants Res 17: 58-66.

15. Lundgren S, Rasmusson L, Sjöström M, Sennerby L (1999) Simultaneous or delayed placement of titanium implants in free autogenous iliac bone grafts. Int J Oral Maxillofac Surg 28: 31-37.

16. Lundgren S, Nyström E, Nilson H, Gunne J, Lindhagen O (1997) Bone grafting to the maxillary sinuses, nasal floor and anterior maxilla in the atrophic edentulous maxilla. A two-stage technique. Int J Oral Maxillofac Surg 26: 428434.

17. Smolka W, Eggensperger N, Carollo V, Ozdoba C, lizuka T (2006) Changes in the volume and density of calvarial split bone grafts after alveolar ridge augmentation. Clin Oral Implant Res 17: 149-155.

18. Barone A, Covani U (2007) Maxillary alveolar ridge reconstruction with nonvascularized autogenous block bone: Clinical results. J Oral Maxillofac Surg 65: 2039-2046.

19. Marx RE, Stevens MR (2010) Atlas of Oral and Extraoral Bone Harvesting Quintessence Publishing Co, Inc, Chicago.

20. Spagnoli DB, Marx RE (2011) Dental implants and the use of rhBMP-2. Oral Maxillofacial Surg Clin North Am 23: 347-361.

21. Yasko AW, Lane JM, Fellinger EJ, Rosen V, Wozney JM, et al. (1992) The healing of segmental bone defects, induced by recombinant human bone morphogenetic protein (rhBMP-2). A radiographic histological, and biomechanical study in rats. J Bone Joint Surg Am 74: 659-670.

22. Sigurdsson TJ, Fu E, Tatakis DN, Rohrer MD, Wikesjö UM (1997) Bone morphogenetic protein-2 for peri-implant bone regeneration and osseointegration. Clin Oral Implants Research 8: 367-374.

23. Fahmy RA, Mahmoud N, Soliman S, Nouh SR, Cunningham L, et al. (2015) Acceleration of alveolar ridge augmentation using a low dose of recombinant human bone morphogenetic protein-2 loaded on resorbable bioactive ceramic J Oral Maxillofac Surg 73: 2257-2272.

24. Ribeiro Filho SA, Francischone CE, de Oliveira JC, Ribeiro LZ, do Prado FZ, et al. (2015) Bone augmentation of the atrophic anterior maxilla for dental implants using rhBMP-2 and titanium mesh: histological and tomographic analysis. Int $\mathrm{J}$ Oral Maxillofac Surg 44: 1492-1498.

25. Misch CM (2011) Bone augmentation of the atrophic posterior mandible for dental implants using rhBMP-2 and titanium mesh: clinical technique and early results. Int J Periodontics Restorative Dent 31: 581-589. 